\section{LA DIMENSIÓN SOCIO ESPACIAL DE LA VIVIENDA RURAL EN LA CIUDAD DE MÉXICO. EL CASO DE LA DELEGACIÓN MILPA ALTA'}

Eduardo Torres Veytia ${ }^{2}$

Luz Malinalli Vega Díaz ${ }^{3}$

Claudia Higuera Meneses ${ }^{4}$

\section{Resumen}

Este trabajo es un avance preliminar de un estudio que se propone analizar, una vez concluido con las acciones de mejoramiento y etapa de evaluación del programa, las condiciones de habitabilidad y calidad de vida, a partir de las características de la vivienda, de los pobladores de la Delegación Milpa Alta en la Ciudad de México. En una primera etapa, el análisis que aquí se presenta, consideramos importante dar a conocer la vivienda que ha sido construida por sus pobladores en Milpa Alta: la organización y distribución espacial e identidades asociadas a la comunidad; es decir, interesa mostrar la vivienda rural que ha sido edificada por los propios habitantes como resultado de las necesidades específicas que material y espacialmente, como en la identidad y percepción, expresan la constitución de un entorno propio y característico de la vivienda rural que requiere considerarse en

\section{SOCIO SPATIAL DIMENSIONS OF RURAL HOUSING IN MEXICO CITY. THE CASE OF MILPA ALTA ${ }^{1}$}

Eduardo Torres Veytia ${ }^{2}$

Luz Malinalli Vega Díaz ${ }^{3}$

Claudia Higuera Meneses ${ }^{4}$

\begin{abstract}
Based on the analysis of housing characteristics, this article is the first part of a study focused on habitability conditions and quality of life of residents from Milpa Alta, Mexico City. In this analysis, it is important to show the type of housing that has been built by people from Milpa Alta, as well as organization, spatial distribution and identities associated to the community; in other words, this paper aims to show rural housing built by their inhabitants as a result of specific needs that express the construction of an environment that should be regarded as a way of life by any instrument of public policy.
\end{abstract}


sus necesidades, problemáticas y diferencias notables como forma de vida en cualquier instrumentación de la política pública.

\section{PALABRAS CLAVE: VIVIENDA, VIVIENDA RURAL, MÉXICO, MILPA ALTA, HABITABILIDAD Y ANÁLISIS SOCIO-ESPACIAL.}

Fecha de recepción: 07.03.11.

Fecha de aceptación: 10.06.11.

1 La investigación se realiza en el contexto del proyecto piloto para el Programa de Mejoramiento de la Vivienda Rural Sustentable (PMVRS) implementado por un grupo de consultores de la Facultad de Arquitectura de la Universidad Nacional Autónoma de México (UNAM) y contratado por el Instituto de Vivienda (INVI) del Distrito Federal en convenio con la Delegación Milpa Alta, para realizar un programa de mejoramiento de la vivienda rural en cuatro poblados de la demarcación y una posterio evaluación de su impacto en las condiciones de vida de los habitantes. Es coordinado por el Arq. Alejandro Suárez Pareyón, profesor y responsable del campo de conocimiento Análisis, Teoría e Historia de la Arquitectura y la Ciudad en el Posgrado de Arquitectura y Urbanismo de la UNAM.

2 México. Licenciado en Antropología Física ENAH-INAH-SEP, Maestro en Antropología Aplicada y Desarrollo Social CIESAS/ Universidad de Sussex y Maestro en Urbanismo Facultad de Arquitectura-UNAM. Profesor e investigador en la Universidad Nacional Autónoma de México UNAM. Correo Electrónico: etowerszone@gmail.com

3 México. Licenciada en Sociología de la Universidad Autónoma Metropolitana. Estudiante Maestría en Urbanismo, del programa de Maestría y Doctorado en Urbanismo en la Universidad Nacional Autónoma de México UNAM. Correo Electrónico: malli. vega@gmail.com

4 México. Licenciada en Sociología y Maestra en Planeación y Políticas Metropolitanas de la Universidad Autónoma MetropolitanaAzcapotzalco. Estudiante doctorado Universidad Nacional Autónoma de MéxicoUNAM.CorreoElectrónico:klausina@gmail.com

\section{KEYWORDS: HOUSING, RURAL HOUSING, MILPA ALTA, HABITABILITY AND SOCIO SPATIAL ANALYSIS}

Received: 07.03.11.

Accepted: 10.06.11.

1 This research is part of the Program for the Improvement of Sustainable Rural Housing; this program was implemented by a group of advisors to the Facultad de Arquitectura at Universidad Nacional Autónoma de Mexico hired by the Federal District's Instituto de la Vivienda and Milpa Alta to develop an improvement program of rural housing an assess the impact of such initiative on inhabitants. This project is coordinated by Alejandro Suárez Pareyón, professor in charge of the post graduate field of knowledge "Analysis, Theory and History of Architecture and City", UNAM.

2 Mexican. BA in Physical Anthropology ENAH-INAH-SEP; MA in Applied Anthropology and Social Development CIESAS, Sussex University; MA in Urban Planning, Facultad de Arquitectura, UNAM. Academic and researcher at Universidad Nacional Autonoma de Mexico UNAM. Email: etowerszone@gmail.com

3 Mexican, BA in Sociology, Universidad Autonoma Metropolitana. Master's student in Urban Planning. UNAM. Email: malli.vega@gmail.com

4 Mexican. BA in Sociology, MA in Planning and Metropolitan Policies. Universidad Autónoma Metropolitana-Azcapotzalco. Doctorate student at UNAM. Email: klausina@gmail.com 


\section{Introducción}

La vivienda rural como tema de estudio y diseño de política pública ha sido poco abordado, la misma investigación que nos ocupa es resultado de esta falta de atención a la demanda y problemática de vivienda para los habitantes de los asentamientos rurales en la Ciudad de México. Desde diversas perspectivas de estudio ${ }^{5}$, esta situación ha sido señalada como parte de un problema de mayor alcance debido a que refiere a la subestimación de necesidades y problemáticas del medio rural, denegación de las condiciones de vida precarias de los habitantes y preeminencia de la problemática del medio urbano. "El estudio de los espacios habitados se orienta casi siempre al examen de las edificaciones y sitios propios de los sectores sociales intermedios o de las clases dominantes. Igualmente se advierte una inclinación de éste hacia las ciudades y, por consiguiente, a descuidar el análisis de la vivienda popular campesina"

Dicha tendencia, de otorgar mayor atención a los problemas urbanos, se ha traducido en una administración y planificación del territorio que invisibiliza el área caracterizada como rural (considerada sin requerimientos para un ordenamiento y

5 Boils Morales, Guillermo, 2003; Flores Rodríguez, Liliana, 2009; Jiménez Rosas, Eric Orlando y Verduzco Chirino, Gemma Luz Sylvia, 2009; Tapia, Ricardo, 2006, Sánchez Quintanar, Concepción, 2006.

6 programación), no obstante del resultado notable del crecimiento y reproducción del asentamiento rural como una solución elaborada por los propios habitantes, pero en un contexto de graves carencias y deficiencias en la utilización de los recursos naturales y el cubrimiento de las necesidades más apremiantes como la vivienda.

Especialmente la vivienda rural como proceso de autoconstrucción se ha subestimado en la problemática y necesidades que representa para el asentamiento rural y condiciones de vida de los habitantes. Estructuras frágiles, carencia en instalaciones sanitarias, en servicios, ubicación en zonas de riesgo, altos costos con gran impacto en la economía familiar durante un largo proceso de edificación, que puede alcanzar hasta 30 años, establecen algunos elementos que indican la falta de atención a los asentamientos rurales y puntualmente a la problemática de la vivienda rural.

En la perspectiva latinoamericana, se comparten varios de los elementos señalados, aun considerando las diferencias en la problemática específica (contexto sociopolítico, económico, cultural, etcétera), como en la intervención de la política pública en el área rural. En la última década (2000-2010) se ha llevado a cabo una importante acción, como política 
habitacional, con la implementación de diferentes programas (erradicación de asentamientos precarios, mejoramiento de la vivienda progresiva, etc.) que plantea una preocupación por conocer el impacto de los programas habitacionales en el medio rural, su concordancia con lo previsto por los planificadores y responsables de la política, sobre todo la efectividad como respuesta a las demandas y expectativas de los habitantes del espacio rural y la compatibilidad con un modelo sustentable para el territorio.

La política habitacional para los asentamientos rurales necesita considerar a la vivienda rural con las características que los habitantes han configurado conforme a las actividades e interacciones sociales, requerimientos y condiciones específicas de una forma de vida. Un tratamiento diferenciado para la vivienda rural resulta imprescindible para instrumentar políticas públicas que efectivamente respondan a sus necesidades, demandas y modo de vida.

En un contexto de desatención y subestimación de la política habitacional para el medio rural resulta necesario estudiar el medio y dar a conocer la vivienda rural actual construida conforme a un contexto sociocultural, territorial y productivo específico; una vivienda rural heterogénea y diversa como el mismo medio y condiciones de vida de los habitantes que demanda una política pública adecuada para la satisfacción de las necesidades

204 revista invi № 73 / Noviembre 2011 / Volumen № 26: 201-223 de mejoramiento y calidad constructiva, sin obviar las funciones estratégicas que desempeñan en la actividad laboral y productiva de los habitantes, respondiendo a la problemática de riesgo y vulnerabilidad e incorporando la dimensión cultural que también es componente imprescindible de la seguridad de la vivienda rural.

La vivienda en general como objeto de estudio requiere analizarse como parte de un sistema socioespacial, integrado por la casa, el asentamiento geográfico y los estilos de vida de sus habitantes ${ }^{7}$. En el caso de la vivienda rural consideramos el tema todavía más demandante debido a la grave problemática de riesgo que presenta, como la desatención de la política pública y desconocimiento del espacio construido por los habitantes en el diseño y operación de algún eventual programa en el medio rural; como se señala para un caso de estudio en Chile, pero plenamente acorde a la situación que se plantea para varios casos en el subcontinente y por supuesto el caso planteado en la Ciudad de México, "no se toma en cuenta el modo de habitar característico, actual, de una familia minera, pescadora, campesina, temporera, obrera forestal, pobladora pescadora ...Todos ellos son genéricamente definidos como 'familias de escasos recursos del sector rural' y los modelos de viviendas son una copia traspasada de los modelos urbanos".

Jiménez y Verduzco, 2009: 46.

Tapia, 2006. 
Destacar la vivienda rural en su organización y distribución espacial, las necesidades como extensión espacial de la familia, flexibilidad del uso y sus funciones en las actividades laborales, la apropiación e identidades asociadas que se analizan en el trabajo, busca aportar en el estudio de la vivienda rural para la consideración de su puntual diseño y construcción, fundamentalmente de la identidad cultural y necesidades de los habitantes de la Ciudad de México, como quienes habitan el área rural de la Delegación Milpa Alta.

Para ello, en la primera parte del trabajo se destacan algunas características socioeconómicas de la población, sucintamente la formación sociohistórica del territorio, conformación de la propiedad social e identidad de los habitantes que han configurado un puntual proceso de poblamiento, lo cual es determinante para conocer la vivienda construida por sus pobladores en Milpa Alta. En tanto, la organización y distribución del espacio se desarrollan en la segunda parte y para finalizar se analiza la necesidad de construir una metodología socio-espacial de la vivienda rural, ahondar en el análisis de su morfología y tipología en la búsqueda de elementos rescatables, que permitan aportar para la atención de la política pública de las necesidades habitaciones de estos pueblos.

\section{La Delegación Milpa Alta en la Ciudad de México}

Se ubica al sureste del Distrito Federal (DF), limita al norte con las delegaciones Tláhuac y Xochimilco, al oeste con Tlalpan y Xochimilco, al este con los municipios de Chalco, Tenango del Aire y Juchitepec del Estado de México y al sur con los municipios de Tlalnepantla y Tepoztlán del Estado de Morelos. Ocupa una superficie total de 28, 375 hectáreas, que representan el $19.06 \%{ }^{9}$ del área total del Distrito Federal y en su totalidad se encuentra catalogada como suelo de conservación y de propiedad social, actualmente el 10\% está ocupada por los núcleos urbanos de las doce comunidades. El 41\% de dicha superficie está dedicada al desarrollo de actividades agropecuarias y en el $49 \%$ restante se encuentran las zonas boscosas.

La Delegación se encuentra ubicada dentro de la Sierra Ajusco Chichinautzin, zona de origen volcánico reciente, en donde los suelos están en proceso de formación. Se considera como una de las regiones de mayor permeabilidad, por lo que es la principal zona de recarga del acuífero de la cuenca de México.

9 INEGI, 2002 


\section{FIGURA 1. LA DELEGACIÓN MILPA ALTA EN LA ZONA METROPOLITANA DEL VALLE DE MÉXICO, 2010.}

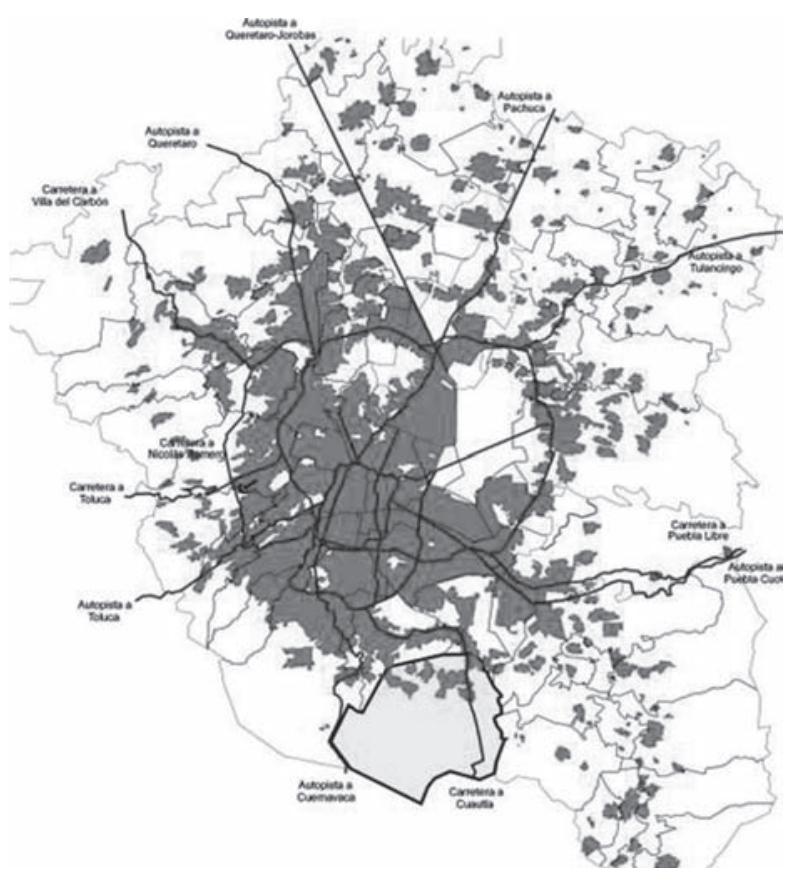

\section{ASPECTOS SOCIOECONÓMICOS DE LA POBLACIÓN}

De acuerdo al II Conteo de Población 2005, realizado por el Instituto Nacional de Estadística y Geografía (INEGI), vivían en Milpa Alta 116 mil personas distribuidas en los doce poblados y en más de cien asentamientos humanos irregulares. De 1950 a 1980 presenta una relativa estabilidad, mientras que entre los años 1980 y 2005 la población se incrementa a poco más del doble. El momento de mayor incremento demográfico para Milpa Alta fue entre 1990 y 2005, duplicando su población a 52,241 habitantes, cifra muy similar a la que tenía en 1980, hasta llegar a la cifra actual.

En la dinámica de poblamiento es de resaltar que el crecimiento natural constituye el componente fundamental de su crecimiento total, posee la tasa global de fecundidad más elevada con 2.53 hijos por mujer, mientras en el DF es de 2.03 (datos 1999), y la mayor proporción de mujeres con tres hijos con respecto al resto de las delegaciones; no solamente se trata de las madres más jóvenes sino también las mujeres con más hijos al final de su vida reproductiva (promedios mayores a los 4 hijos entre las mujeres del grupo de edad 45-49 años), en donde el primer hijo lo alcanzan cuando tienen entre 20 y 24 años; el segundo entre los 25 y 29 años; el tercero y cuarto hijo entre los 45 y 49 años.

Y si se toma en cuenta la estructura por edad de la población que es predominantemente joven (edad mediana de 24 años, la más baja del DF), evidentemente se mantendrá una fuerte presión en la demanda de vivienda, equipamientos y servicios debido al incremento en la formación de nuevos hogares, que son los más grandes en promedio con 4.15 miembros por hogar y 3.67 en el DF.

En esta dinámica de poblamiento, los inmigrantes han tenido un impacto menor, representaron poco 
menos del 1\% de la población y el 5\% del total de 19,122 personas en que aumentó la población de la Delegación entre 2000 y 2005. En síntesis, es la Delegación con el mayor porcentaje de habitantes que hablan una lengua indígena con respecto a su población, tiene los menores niveles de población con educación superior y la mayor cantidad de gente que gana menos de dos salarios mínimos y la menor de aquellos que ganan más de 10 salarios mínimos.

\section{El territorio de Milpa Alta}

Son nueve poblados rurales los que se reconocen como fundadores del antiguo "Malacachtepec Momozco", a partir de lo cual se han autonombrado "Confederación de los Nueve Pueblos de Milpa Alta"10, que son Villa Milpa Alta, San Juan Tepenáhuac, San Francisco Tecoxpa, San Pablo Oztotepec, San Pedro Atocpan, Santa Ana Tlacotenco, San Jerónimo Miacatlán y San Lorenzo Tlacoyucan. El caso del poblado de San Agustín Ohtenco fue fundado por la migración de un barrio de Villa Milpa Alta y por ende forma parte de la integración colonial de Milpa Alta y del reconocimiento de la

10 Copia Certificada de documentos relativos a la comunidad de Milpa Alta. Delegación Milpa Alta, Distrito Federal, expedida a solicitud del C. Julián Flores Aguilar, representante de bienes generales de dicho lugar, 27 de junio de 2002.

\section{GRÁFICO 1. CRECIMIENTO DE POBLACIÓN CENSAL EN LA DELEGACIÓN MILPA ALTA, 1950-2005.}

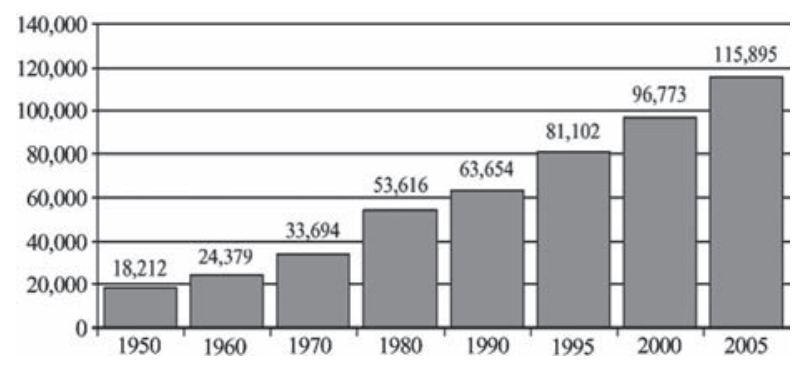

propiedad de la tierra por parte de los españoles ${ }^{11}$. Hay dos poblados que se identifican como de procedencia xochimilca: San Salvador Cuauhtenco y San Bartolomé Xicomulco. En cuanto al poblado de San Antonio Tecómitl, tiene un origen relacionado con las comunidades Tláhuicas.

Con base en este relato histórico se establece el sentido de pertenencia de los habitantes, porque el territorio les pertenece histórica, social y jurídicamente, como se demuestra en el régimen de propiedad social y el derecho absoluto sobre la tierra de todos los pobladores originarios.

11 Archivo General de la Nación. Grupo Documental Tierras, Vol. 3032, exp. 3, fs 202-206v., 217-218v. y 227-227v. (Santa Marta Xocotepetlalpan).

revista invi № 73 / Noviembre 2011 / Volumen Nº 26: 201-223 207 


\section{LA FORMACIÓN SOCIO-HISTÓRICA DE UNA IDENTIDAD: LA PROPIEDAD SOCIAL DEL TERRITORIO}

Los nueve poblados rurales cuentan con un documento rector que hace posible su unidad, los Títulos Primordiales, que constituyen la base del ordenamiento jurídico que regula la propiedad comunal con una superficie total de 26,913.6 hectáreas, estos son considerados en el Artículo 27 Constitucional, su reglamentación en la Ley Agraria y otras leyes, códigos y reglamentos sobre la materia. Conjuntamente está la propiedad ejidal del territorio que se establece el 4 de octubre de 1925. Los pobladores originarios de Santa Ana Tlacotenco, San Francisco Tecoxpa, San Jerónimo Miacatlán y San Juan Tepenáhuac presentaron ante la Comisión Agraria la solicitud formal de restitución de tierras.

La posesión definitiva se dio el 3 de marzo de 1930, la superficie dotada fue de 519 hectáreas, cantidad insuficiente para permitir la subsistencia plena de sus habitantes. En la actualidad el ejido cuenta con un total de 1,892 hectáreas y mantiene la actividad productiva. Se destaca la problemática que enfrentan por la falta de apoyos a proyectos productivos que hasta el momento son insuficientes.

\section{FIGURA 2. PROPIEDAD SOCIAL DEL TERRITORIO DE LA DELEGACIÓN MILPA ALTA.}



\section{LOS CUATRO POBLADOS RURALES}

Algunos de estos pueblos se han ido uniendo entre sí a partir de los procesos de desdoblamiento natural que existen en el territorio. En el caso de los cuatro pueblos centrales donde se realiza el proyecto piloto para el Programa de Mejoramiento de la Vivienda Rural Sustentable, San Agustín Ohtenco, San Francisco Tecoxpa, San Jerónimo Miacatlán y San Juan Tepenáhuac, los tres primeros prácticamente están articulándose entre sí. Tepenáhuac, por su lado, sigue siendo independiente, pero dentro de poco puede ser parte de este crecimiento hacia el centro de la Delegación. 


\section{FIGURA 3. ÁREA CENTRAL DE LA DELEGACIÓN MILPA ALTA 2010: POBLADOS DE SAN AGUSTÍN OHTENCO, SAN FRANCISCO TECOXPA, SAN JERÓNIMO MIACATLÁN Y SAN JUAN TEPENÁHUAC.}

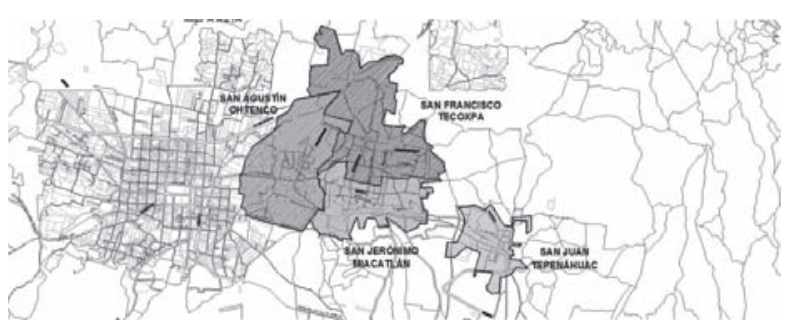

\section{CUADRO 1. SUPERFICIE DE LOS CASCOS URBANOS} DE LOS 4 POBLADOS RURALES.

\begin{tabular}{lc}
\hline Poblados Rurales & Área en Ha \\
\hline San Juan Tepenáhuac & 43.00 \\
\hline San Francisco Tecoxpa & 93.52 \\
\hline San Agustín Ohtenco & 78.67 \\
\hline San Jerónimo Miacatlán & 51.38 \\
\hline
\end{tabular}

En 2005 vivían un poco más de 10 mil personas distribuidas en los cuatro poblados rurales y en nueve asentamientos irregulares. El crecimiento de la población en los cuatro poblados rurales, asî como el crecimiento de número de viviendas, se describe a continuación.

La estructura agraria-rural y sus distintas fases del proceso son las actividades económicas por excelencia de las comunidades, pero desde 1990 se ha desarrollado un proceso de incorporación del comercio al por menor y la venta de productos alimenticios, como alternativas para obtener ingresos familiares. Y no obstante que la participación de la población en las actividades relacionadas con la producción agrícola ha disminuido sensiblemente, al final de la década 1990 a 2000 Milpa Alta se ha mantenido como el principal abastecedor de nopal (cactácea comestible) en la ciudad (80\% de la demanda)

\section{CUADRO 2. PRINCIPALES CULTIVOS EN 2004.}

\begin{tabular}{lcc}
$\begin{array}{l}2004 \text { Cultivo } \\
\text { Principal }\end{array}$ & $\begin{array}{c}\text { Superficie Sembrada } \\
\text { Milpa Alta }\end{array}$ & $\begin{array}{c}\text { Superficie } \\
\text { Sembrada DF }\end{array}$ \\
\hline Avena Forrajera & 1586 & 8325 \\
\hline Maíz Grano & 2910.2 & 7224 \\
\hline Nopal Verdura & 4159.3 & 4159.3 \\
\hline Total Milpa Alta & 9129.3 & 25839.2 \\
\hline
\end{tabular}

Para 2004 son tres cultivos los más relevantes: el nopal que corresponde al 100 \% de la producción; el maíz grano como importante en la Delegación y la avena forrajera en cuanto a la superficie sembrada. Así se observa que el cultivo más importante en Milpa Alta, en cuanto a su superficie sembrada, volumen y valor de la producción, es el nopal, por ello se muestra a continuación un cuadro con la distribución de superficie sembrada para diversos poblados rurales. 
CUADRO 3. POBLACIÓN, VIVIENDA Y HABITANTES POR VIVIENDA.

\begin{tabular}{|c|c|c|c|c|c|c|c|c|c|c|c|c|}
\hline \multirow[t]{2}{*}{ Poblados Rurales } & \multicolumn{4}{|c|}{ Población } & \multicolumn{4}{|c|}{$\begin{array}{l}\text { Viviendas Particulares } \\
\text { Habitadas }\end{array}$} & \multicolumn{4}{|c|}{ Hab/Viv } \\
\hline & 1990 & 1995 & 2000 & 2005 & 1990 & 1995 & 2000 & 2005 & 1990 & 1995 & 2000 & 2005 \\
\hline \multirow{4}{*}{$\begin{array}{l}\text { San Agustín Ohtenco, } \\
\text { San Francisco Tecoxpa, } \\
\text { San Jerónimo Miacatlán } \\
\text { y San Juan Tepenáhuac }\end{array}$} & 2,002 & 2,661 & 3,056 & 3,620 & 374 & 549 & 638 & 823 & 5.35 & 4.85 & 4.79 & 4.40 \\
\hline & 1,483 & 1,831 & 2,037 & 2,413 & 274 & 396 & 436 & 548 & 5.41 & 4.62 & 4.67 & 4.40 \\
\hline & 1,732 & 1,806 & 2,396 & 2,839 & 321 & 382 & 517 & 645 & 5.40 & 4.73 & 4.63 & 4.40 \\
\hline & 605 & 793 & 977 & 1,157 & 125 & 185 & 240 & 263 & 4.84 & 4.29 & 4.07 & 4.40 \\
\hline TOTAL & 5,822 & 7,091 & 8,466 & 10,030 & 1,094 & 1,512 & 1,831 & 2,280 & 5.32 & 4.69 & 4.62 & 4.40 \\
\hline
\end{tabular}

\section{CUADRO 4. DISTRIBUCIÓN DE LA SUPERFICIE CULTIVADA DE NOPAL.}

\begin{tabular}{lc}
2004 Poblado & Superficie Cultivada ha. \\
\hline San Juan Tepenáhuac & 138 \\
\hline San Francisco Tecoxpa & 144 \\
\hline San Agustín Ohtenco & 174 \\
\hline San Jerónimo Miacatlán & 198 \\
\hline Total Milpa Alta & 6000 \\
\hline
\end{tabular}

Las modificaciones socioeconómicas y culturales de la población (urbanización del territorio, transformación del sistema agrícola, repunte de la producción de alimentos, el comercio y los servicios, reorganización del movimiento comunero) han reforzado el interés de los habitantes por conservar la tierra de cultivo. Si bien se proponen participar de las ventajas del desarrollo urbano, esto es sin renunciar a su identidad como pueblo originario y de acuerdo a una visión propia de este desarrollo. La organiza-

210 revista invi № 73 / Noviembre 2011 / Volumen № 26: 201-223 ción en mayordomías en cada poblado son de los elementos que refuerzan las tradiciones que distinguen el modo de vida. Es la manera como se conforma una importante dimensión de su cultura, el culto a los santos asociados a la religiosidad prehispánica y su vinculación con la propiedad de la tierra.

\section{La vivienda}

Hasta mediados del siglo XX los espacios fundacionales de los poblados rurales de Milpa Alta se constituían por una arquitectura vernácula de la región sur y sureste de la cuenca, caracterizada por construcciones habitacionales con muros de piedra volcánica y techumbres a dos aguas cubiertas con teja plana llamada "marsellesa", pero la expansión del área urbana de la ciudad y el notable crecimiento demográfico de la segunda mitad del siglo XX ha marcado la morfología del espacio rural y urbano. 
Como varios autores lo han señalado, "es difícil que las viviendas del campo conserven sus rasgos y procedimientos tradicionales, pues la intensa presión publicitaria que desde los grandes centros de población se emite hacia las áreas campesinas ha ejercido una gran influencia de cambio"12.

Si bien el paisaje dominante en la Delegación Milpa Alta es la construcción y mantenimiento de un complejo sistema agrícola cuyo vestigio son las terrazas ahora mayormente sembradas de nopal, la estructura urbana de Milpa Alta se caracteriza por un espacio habitacional unifamiliar, la mayoría son viviendas propias de uno o dos pisos y distintos grados de consolidación por el proceso de autoconstrucción imperante en la edificación de las viviendas, aunque se ha vuelto común la construcción de vivienda por contrato, lo cual ha conformado un panorama arquitectónico de los poblados rurales que combina elementos tradicionales y modernos. En las últimas décadas se ha generado un tipo de construcción que refleja la intención de adoptar un estilo de vida similar al que se desarrolla en las zonas más céntricas de la Ciudad de México. De hecho ahora se observan viviendas de dos plantas con varias habitaciones y construidas con materiales de block, tabicón, sustituyendo a la piedra y el adobe.

Contradictoriamente con su localización geográfica y el paso de una importante carretera de ni-

12 Boils, 2003: 43 vel nacional, Milpa Alta es una Delegación poco comunicada al resto de la ciudad; se debe mencionar que la movilización hacia otras zonas de la ciudad a partir de los poblados rurales de Milpa Alta, puede llegar a tener un alto costo en términos económicos y de tiempo debido a las pocas vías de conexión que se dispone.

En este contexto, uno de los aspectos importantes en este territorio en particular es la vivienda. Como sucede en la mayor parte de la ciudad, es un bien común, no sólo porque sirve para guarecerse de las inclemencias del tiempo, sino que a su vez denota un patrimonio. Por un lado, de manera individual, pero también colectivamente para la familia. Además, en comparación de otros pueblos en la ciudad, la tierra sigue teniendo en esta demarcación una connotación importante: es el sustrato socio-histórico de la identidad de los habitantes. Es un elemento determinante a considerar en cualquier gestión del territorio, más cuando la vivienda comparte este espacio contiguo con el campo —-todavía laborable_, la milpa.

\section{IDENTIDAD (PERTENENCIA, ARRAIGO, TERRITORIALIDAD)}

En gran medida las viviendas están asentadas en lo que antiguamente fue un campo de cultivo, que a partir de las relaciones de parentesco se dividió y 
cedió a los hijos; es el proceso de poblamiento que de generación en generación se ha ido dando en este espacio de la ciudad.

A partir de esta cesión de derechos de la tierra, los pobladores han ido autoconstruyendo su espacio habitable, es decir, una producción social del espacio; es un caso particular en comparación a otros pueblos que pueden compartir escenarios similares, porque la especificidad está en el arraigo a la tierra, las tradiciones, los usos y costumbres en Milpa Alta que componen el reconocimiento de sí mismos como propietarios del territorio. Es determinante conocer y tener en cuenta en esta delegación de la Ciudad de México, que sus habitantes tienen una visión (es decir, un imaginario colectivo) distinto a los habitantes del resto de la ciudad. En este sentido, la pertenencia al pueblo es más significativa que a la demarcación delegacional, aún cuando la referencia también podría ser a Milpa Alta, pero eso habla de prácticas sociales y culturales muy arraigadas al pueblo, y también a la delegación, que en cierto sentido los distancia de lo que es la Ciudad de México en sí.

Puntualmente, el vínculo con la tierra configura un territorio específico cuya geografía simbólica se remonta a la época colonial, en la cual se produce una "reestructuración étnica" de los grupos indios después del enfrentamiento con los representantes

212 revista invi № 73 / Noviembre 2011 / Volumen N²6: 201-223 de la iglesia católica y de la dominación española ${ }^{13}$, en la que se fortalecen las bases de su identidad: la defensa del territorio y la conservación del mismo, como procesos socio-históricos que hasta la fecha tienen impacto en diversos espacios de la vida comunitaria, desde festividades, tradiciones, usos y costumbres hasta la elección de autoridades comunitarias (coordinador de enlace territorial por cada poblado, representantes comunales y ejidales, etc.), gestión del territorio y el espacio más privilegiado de esas prácticas, es decir, la casa-habitación.

\section{LOS PRIMEROS RESULTADOS}

De los resultados alcanzados hasta el momento en dicha investigación, referentes a los datos estadísticos de 268 casos procesados (la muestra comprenderá 674 casos en total), es posible señalar que el $66.15 \%$ de los solicitantes tiene de 20 a 40 años de edad, de los cuales el $26.15 \%$ solicita crédito para vivienda nueva y el $40 \%$ para mejoramiento de vivienda. Se trata de familias que están iniciando la construcción de la vivienda, desde los que recién la forman (20 años con 1 hijo), hasta los que están pasando del cuarto redondo a la construcción de 1 a 3 cuartos (30 años con dos hijos o más). El proceso de autoconstrucción de la vivienda abarcaría las dos primeras etapas, cuando se edifica la cocina, el baño y una recámara; de ahí que el 40\% de las solicitudes para mejoramiento de vivienda se

13 Duhau, 2008: 364

ARTíCULO: La dimensión socio espacial de la vivienda rural en la Ciudad de México. El caso de la Delegación Milpa Alta / Eduardo Torres Veytia, Luz Malinalli Vega Díaz y Claudia Higuera Meneses 
refieran al "echado de la losa", la construcción de muros de tabicón en cocina, recámara, sala y baño, así como mejora de las condiciones de ventilación e iluminación.

Contrario a lo que se podría suponer, el crédito de mejoramiento de vivienda, en el caso de los habitantes de la Delegación Milpa Alta, se aplica para conformar las condiciones de habitabilidad de la vivienda y no para la consolidación de la misma. Esta última etapa se refiere al 33.85\% del resto de los solicitantes con edad de 41 a 68 años, de los cuales $3.08 \%$ solicita crédito para vivienda nueva y $30.77 \%$ para mejoramiento de vivienda que considera la aplicación en acabados (aplanados, impermeabilización, puertas, ventanas, recubrimientos, muebles para baño, etc.).

Como se observará en el siguiente apartado, en el largo proceso de autoconstrucción de la vivienda la disponibilidad de los servicios ocupa un lugar relevante para la habilitación del uso de los espacios. En estos primeros resultados, el 69.23\% cuenta con abastecimiento de agua potable y drenaje, el 52.31\% dispone del servicio de electricidad con medidor y el $47.69 \%$ lo hace sin medidor.

En cuanto a la ocupación del predio, el 35.38\% corresponde a la categoría unifamiliar, es decir, una sola familia ocupa el mismo predio; el número de familias ocupantes por predio varía respecto a la categoría plurifamiliar: el 24.61\%, de 2 a 3 familias, ocupan un predio con dos viviendas y el
$10.77 \%$, de 4 a 8 familias, ocupan un predio con 3 viviendas.

Las condiciones económicas referidas al ingreso monetario, señala que el $63.08 \%$ de los solicitantes tiene ingresos familiares de 2,000 a 6,000 pesos mexicanos al mes, el cual se integra con el jornal de todos los integrantes de la familia que se van incorporando a la edad de trabajar en actividades como faena en el campo, limpieza de nopal, comercio y su venta, chofer, albañilería, empleado en tienda de abarrotes, en restaurantes, limpieza, etc. La economía familiar se sustenta en el trabajo conjunto en el campo, el comercio y los servicios. El $36.92 \%$ de los solicitantes que indica como ingreso familiar más de 6,000 hasta 13, 127 pesos mensuales, se ocupa en las actividades mencionadas en el párrafo anterior y en otras más calificadas como contador, enfermero y técnico en informática.

Hasta aquí, algunos de los datos estadísticos de la situación social, económica y habitacional de las familias solicitantes de crédito para el mejoramiento de vivienda en la delegación Milpa Alta. Esto apunta hacia algunos elementos de acercamiento a la problemática de la vivienda, que se desarrolla en el siguiente apartado complementando con información cualitativa, resultado del procesamiento de trabajo de campo hasta ahora realizado por medio de entrevistas, observación participante y mapas mentales, principalmente. 


\section{LA VIVIENDA CONSTRUIDA POR SUS POBLADORES EN MILPA ALTA}

La vivienda per sé es el espacio donde se materializan, literalmente, parte de los comportamientos, prácticas e identidades que diferencian a los habitantes de Milpa Alta. En primer término, evidencia el reconocimiento por la comunidad como habitantes originarios, es difícil tener acceso a la tierra como avecindados (expresión utilizada para denominar a los habitantes que no nacieron en Milpa Alta) y en consecuencia a la infraestructura y equipamiento urbano. Por supuesto que sucede como parte de un mercado de suelo irregular, pero este acceso a la tierra marca un proceso de construcción y consolidación de la vivienda aún más largo y difícil.

En segundo lugar, más en paralelo, determina la gestión del territorio en general, no sólo en cuanto a la introducción de los servicios básicos como agua, drenaje, electricidad, etc., también en la integración a las actividades que delimitan la forma de vida de los habitantes, es decir, la participación en las festividades, actos religiosos, procesiones y demás ceremonias con motivo de la llegada del Santo Patrono u otro suceso conmemorativo importante, lo cual establece la pertenencia y el lugar que se ocupa en la comunidad misma. En conjunto condiciona el acceso a cualquier tipo de apoyo y programa, ya sea la construcción y pavimentación de caminos de acceso, como actividades domésticas y productivas de las familias.

214 revista invi № 73 / Noviembre 2011 / Volumen № 26: 201-223
En este contexto, parecería que la ocupación del suelo por los habitantes originarios (comúnmente el momento que los padres heredan la tierra a los hijos es al formar una nueva familia) resuelve la problemática de acceso al suelo, pero esto no sólo es incierto sino que además lleva a subestimar la problemática de la vivienda rural, porque se infiere que la garantía de contar con suelo para vivienda soluciona en automático sus necesidades al respecto.

Hasta ahora, por los resultados de la investigación, es posible precisar que acontece lo contrario. La indagación del proceso de autoconstrucción permite detectar graves carencias y necesidades que son ignoradas como problemática de la vivienda rural. Efectivamente el acceso al suelo, a través de la herencia familiar, posibilita contar con un espacio habitacional para dar forma al proyecto de vida de formar una familia (en gran parte sin tener que sufragar los gastos de un enlace civil), lo que permite concretar la unión de la pareja. Hasta ahí, cumple con la realización de parte de sus expectativas de vida, sin embargo, en la mayoría de los casos, se materializan en un cuarto redondo.

Literalmente, se puede señalar que la base de la vivienda rural son sus cimientos, a partir de lo cual se desarrolla la edificación, por lo que se podría considerar efectivamente una solución a las necesidades de vivienda de estos habitantes, si no fuera porque esta edificación constituye un proceso de 30 años que absorbe gran parte del presupuesto 
familiar, representa condiciones de inhabitabilidad, hacinamiento, carencia de servicios y detrimento de las condiciones de vida.

No sólo el largo proceso de edificación de la vivienda rural pasa "desapercibido", en consecuencia tampoco se contemplan las diferentes etapas del proceso de autoconstrucción porque del cuarto redondo a la construcción de 2 o más cuartos, que permita a la familia contar con una cocina, baño y recámara, transcurren de 5 a 10 años. Un tiempo durante el cual la familia, para entonces ya integrada además de los jóvenes padres por dos hijos pequeños, ocupa un cuarto donde realiza la totalidad de las labores domésticas que van desde cocinar hasta dormir. Las condiciones de iluminación y ventilación son mínimas, en ocasiones tiene acabados que hacen más accesible el espacio, pero no elimina los problemas de hacinamiento e infuncionalidad del mismo.

"Se ocupa... pero no se vive bien", a decir de uno de los habitantes entrevistados, porque además está la cuestión de compartir los servicios (drenaje, agua, electricidad) con la familia "titular" a veces paterna y otras materna. Depende si es la madre o el padre quien hereda la tierra y lleva a cabo su lotificación para repartir a los hijos, por lo que no estamos hablando de una familia extendida sino de varias, de 3 hasta 8 familias en promedio,

ARTÍCULO: La dimensión socio espacial de la vivienda rural en la Ciudad de México. El caso de la Delegación Milpa Alta / Eduardo Torres Veytia, Luz Malinalli Vega Díaz y Claudia Higuera Meneses que ocupan un mismo predio, conviviendo en el espacio habitacional de la familia "titular" donde comparten y negocian día a día el uso de espacios como la cocina y el baño, principalmente.

El resultado varía en cuanto al impacto en la convivencia familiar y generación de conflictos, si bien la expectativa es la misma: la construcción de la propia vivienda, que se realiza con un costo muy alto para las posibilidades de la economía de los habitantes de Milpa Alta, basada en gran medida en el trabajo en el campo, pequeño comercio y servicios que forman el ingreso familiar y representan en promedio ingresos de 2,000 a 6,000 pesos mexicanos al mes ${ }^{14}$ y sin seguridad social, debido a que se trata de actividades catalogadas por cuenta propia.

De ahí el largo proceso de autoconstrucción en tanto que absorbe en gran medida el presupuesto familiar, por lo que transcurren grandes periodos entre una etapa y otra, entre el cuarto redondo y la vivienda con baño, cocina y una recámara, entre la vivienda en obra negra y la vivienda con acabados y servicios.

Como resulta evidente, los habitantes de Milpa Alta atienden estas necesidades con sus propios recursos; son pocos los programas federales y estatales que han operado en el territorio y sólo a través de algunos apoyos específicos. Más bien se subestima la problemática habitacional por catalogarse

14 De 170.251 a 510.741 dólares. 
como área rural y que cada familia logra resolver en el acceso a la tierra.

Esta situación, desde luego se resuelve, pero con graves dificultades. El proceso de autoconstrucción resulta más bien la única alternativa posible para los habitantes de Milpa Alta, lo cual no significa que se lleve a cabo en las mejores condiciones para atender las necesidades de las familias y especificidades de la problemática del territorio ${ }^{15}$, esto se logra después de un largo tiempo y queda a cargo de los mismos habitantes de Milpa Alta.

En la solución constructiva, que se desarrolla en la segunda etapa del proceso de autoconstrucción, se calcula la recámara como prioridad y la cocina. El baño se delimita con la colocación del inodoro pero se mantiene en obra negra, sin lavabo y en algunos casos con regadera. Lo fundamental es la edificación de los cuartos con techo de concreto y muros de tabicón; es lo que finalmente hace perceptible, para los habitantes, la edificación de su vivienda junto con la independencia de la familia "titular", en su caso de las demás familias del predio. Otorga forma a la vivienda aun con características constructivas poco elaboradas, dimensiones reducidas y condiciones de inhabitabilidad.

El sufragar el costo total de la vivienda rural, por supuesto materiales industriales como el tabicón y la losa que tienen un precio elevado, de 2 a 3 tra-

15 Sánchez Quintanar, Concepción, 2006.

216 revista invi № 73 / Noviembre 2011 / Volumen № 26: 201-223 bajadores y demás gastos que pueden oscilar desde la limpieza del terreno hasta su cimentación y nivelación, entre otros, limitan la continuidad de la construcción por lo que la vivienda se mantiene en estas condiciones no menos de 5 años.

Durante este tiempo se priorizan otros gastos en el presupuesto familiar enfocados a la manutención y educación de los hijos, aumento en el tamaño de la familia (es de recordar que los hogares de Milpa Alta son los más grandes del DF), que no sólo detiene la construcción de la vivienda sino que la mantiene con graves carencias: limitadas fuentes de iluminación natural hacia el interior de la vivienda, la falta de instalaciones sanitarias por la carencia de servicios que posterga la construcción del baño, el cual se coloca fuera del espacio destinado para la vivienda, desprotección del medio natural. En Milpa Alta (dada su localización geográfica) el invierno es particularmente crudo, así que una vivienda en obra negra, con un número variable de ventanas, pero sin vidrios y herrería, tiene poca adecuación climática a las inclemencias del tiempo.

Para la tercera etapa, se construyen el resto de los cuartos que darán forma a la vivienda rural como tal, esto es, recámaras para los hijos, los padres, cocina, sala, comedor y finalmente el baño con instalaciones sanitarias, aunado a que se conserva el espacio para lavar y las actividades productivas, 
regularmente limpieza del nopal, mantenimiento de animales de corral para aprovechar los desechos como abono, bodega para guardar materiales y demás herramientas. Es de destacar que se enfatiza, independientemente de la nopalera, la presencia de árboles, plantas y flores en el área contigua a la vivienda, que también se llega a utilizar como sitio de descanso.

En promedio, los habitantes de Milpa Alta logran edificar una vivienda con estas características en el transcurso de 20 años. La estructura de la edificación es la parte consolidada en una vivienda de dos niveles, cuyo segundo nivel se mantiene en obra negra, donde se incorpora la escalera, interior o exterior, comúnmente en condiciones inseguras con material frágil, sin herrería ni protecciones. Para entonces, ya se observan las diferencias en el gusto por las propuestas arquitectónicas, si bien gran parte de las modificaciones operadas en las construcciones se realizan hasta una cuarta etapa, cuando finalmente se logra invertir en los acabados de la vivienda: aplanados, impermeabilización, puertas y ventanas, muebles para baño, recubrimiento en pisos, en muros y plafones, instalación eléctrica, hidráulica, sanitaria, entre otros.

El proceso de autoconstrucción, por supuesto, no es lineal y no siempre se llega a constituir una vivienda con los acabados básicos, regularmente se mantiene una parte consolidada y la otra permanece en obra negra. El proceso de consolidación llega a superar los 30 años, no obstante resulta lo único accesible a la economía de sus habitantes, dada la falta de atención por la política habitacional de los organismos públicos, que se adecue a las necesidades y especificidades de la problemática de la vivienda rural en Milpa Alta.

\section{ORGANIZACIÓN Y DISTRIBUCIÓN ESPACIAL (USO DEL ESPACIO Y CONFORMACIÓN DE LO HABITABLE)}

La vivienda se organiza a partir de dos espacios en particular, la cocina y el dormitorio. La cocina es el eje motor de la vivienda, que por lo general está contiguo al comedor. En comparación a las viviendas en zonas residenciales altas, que tienen una estancia o sala para el encuentro y convivencia, en el caso de la vivienda rural en Milpa Alta, este espacio termina siendo la cocina, debido a que muchas de las viviendas en el proceso de autoconstrucción se forman desde un cuarto-redondo, y a partir de dos cuartos (son tres, contando el baño, pero son pocas las familias que lo incluyen hacia su interior, en gran medida se sigue conservando al exterior) el espacio prioritario que se conforma es la cocina, que es determinante para atender gran parte de las actividades domésticas, donde se desarrolla primordialmente la convivencia familiar y es el espacio exterior de la familia, el contacto con la comunidad, ahí se reciben a las amistades, a los familiares y cualquier visita. Conforme se va produciendo 
FOTOGRAFÍA 1. VIVIENDA RURAL AUTOCONSTRUIDA.

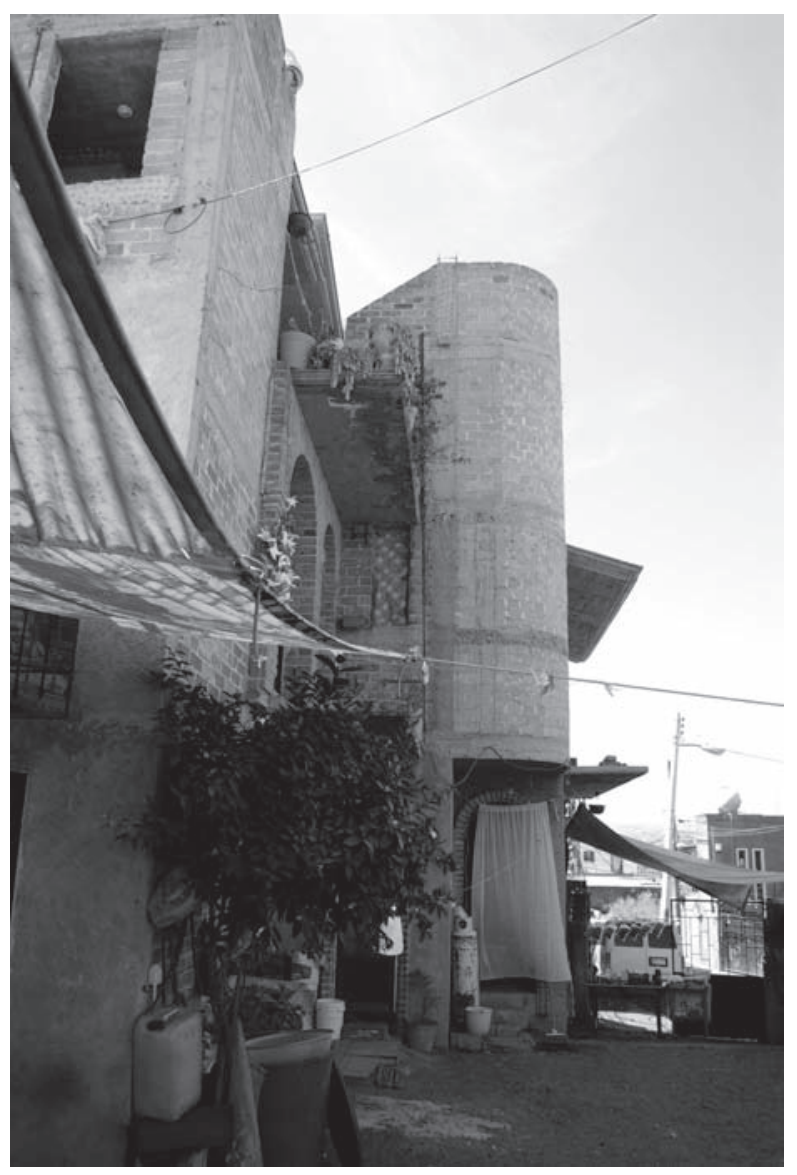

218 revista invi № 73 / Noviembre 2011 / Volumen № 26: 201-223
FOTOGRAFÍA 2. LA COCINA COMO UN EJE DE IDENTIDAD.

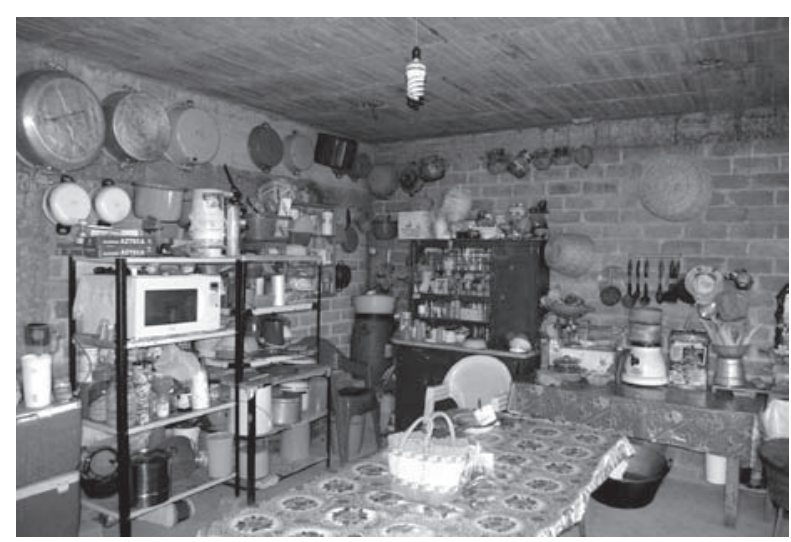

FOTOGRAFÍA 3. DIVERSIDAD EN LOS ESPACIOS.



ARTíCULO: La dimensión socio espacial de la vivienda rural en la Ciudad de México. El caso de la Delegación Milpa Alta / Eduardo Torres Veytia, Luz Malinalli Vega Díaz y Claudia Higuera Meneses 
FOTOGRAFÍA 4. USOS Y FUNCIONES DE LA VIVIENDA RURAL.



la vivienda, y añadiéndole cuartos (habitaciones) a la misma, van surgiendo otros espacios para hacer diferentes actividades como las descritas.

Es el caso de la estancia como tal, un espacio que termina siendo la sala, contigua al comedor, es decir, no ocupa un lugar específico, es movible, alternado y siempre sustituible. El espacio existe por los objetos que en el mismo se colocan, como fotos familiares, trofeos deportivos, televisión, equipo de sonido, etcétera, pero en cualquier momento se le coloca material de trabajo, cajas, herramientas, aunque también es el espacio donde se puede llegar a interactuar con los habitantes de la vivienda. La cocina es el comedor, estancia, sala (más allá de

ARTíCULO: La dimensión socio espacial de la vivienda rural en la Ciudad de México. El caso de la Delegación Milpa Alta / Eduardo Torres Veytia, Luz Malinalli Vega Díaz y Claudia Higuera Meneses ser el espacio donde pasan más tiempo en el día), es el lugar privilegiado para entender como funciona la familia y la vivienda, a partir de la cocina se organiza el modo de vida de sus habitantes. La colocación de los trastos, ollas, electrodomésticos y demás aparatos que sí tienen una función, establece la organización de la actividad de la familia.

Otro espacio relevante en gran parte de estas viviendas, es el dedicado a la actividad laboral, en algunas viviendas es el local comercial, en otras es el espacio para el cultivo, donde se tienen los granos, también se prepara el "relleno" (un abono natural hecho a partir de las sobras del nopal), y el lugar donde se procesa el nopal. 


\section{CONSTRUYENDO UNA METODOLOGÍA SOCIO- ESPACIAL DE LA VIVIENDA RURAL EN MILPA ALTA}

Uno de los factores importantes en el estudio de la vivienda rural, y en particular de Milpa Alta, es poder comprender y entender lo que pasa en su interior, así como al exterior de la misma. Por tal motivo, no sólo es relevante conocer la conformación de la vivienda en sí, es decir no sólo la tipología, sino también haciendo la relación de ésta con el contexto en el que está inmersa, esto es, la morfología de un asentamiento rural, en este caso del pueblo. Es esencial conocer los materiales con los cuales están hechas, sobre todo observar a la vivienda como una unidad que pueda ser entendida en el conjunto de un todo, sin por ello perder su especificidad. Asimismo, esta metodología implica no sólo referencias a lo urbano-arquitectónico, sino a otros campos en donde las ciencias sociales tienen mucho mayor campo desarrollado en el estudio e investigación de la vivienda rural. En esta sección se esbozarán cada uno de los componentes que consideramos necesarios para la conformación de una metodología en particular.

\section{LA VIVIENDA RURAL (MORFOLOGÍA Y TIPOLOGÍA)}

La vivienda rural en la Ciudad de México tiene diferentes tipologías y presenta una morfología urbana que está inmersa en diferentes contextos y realidades sociales, en tanto que la mayor parte del área urbana en algún tiempo fueron pueblos rurales dedicados a la agricultura y ganadería. En Milpa Alta encontramos una variedad significativa en relación a los tipos de vivienda, por un lado se encuentra la vivienda vernácula, pero son pocas las que se conservan como tal; la vivienda vernácula transformada, que en términos de porcentaje prácticamente el $60 \%$ de las viviendas que son patrimoniales en estos cuatro pueblos se han transformado a través del tiempo; y por otro lado están las viviendas que se han edificado en los terrenos contiguos a las casas de los padres o a veces en otros terrenos donde se encuentra la milpa, pero que se ubican hacia el interior del casco urbano permitido y delimitado por las normas urbanas.

Además, esta variedad de viviendas en la actualidad ha sufrido transformaciones importantes, no sólo en la tipología exterior, sino también al interior, a veces resultante de copias de modelos de casas que se llegan a ver en los medios masivos (televisión, Internet o revistas), o en los viajes al resto de la ciudad. En gran medida quienes se dedican al comercio del nopal realizan varios traslados hacia otros puntos de la ciudad y de la zona metropolitana.

La influencia "arquitectónica" se observa en otros modelos de vivienda, que emulan el tipo residencial y hasta monumental. Así como existe el "sueño americano", en la Ciudad de México podría existir el "sueño del capitalino" como un 
modelo a seguir, es así que van surgiendo espacios que imitan a las viviendas ubicadas en las zonas residenciales de delegaciones como Coyoacán, Tlalpan, pero sin tener una significación en particular. Viviendas que se convierten en espacios que a largo plazo terminan siendo obsoletos, porque en la vida cotidiana no se utilizan.

Un elemento que también ha apoyado esta influencia es la relación con las delegaciones limítrofes, especialmente a través de un punto como el pueblo de San Antonio Tecómitl que está prácticamente unido a los pueblos de la delegación Tlahuac (San Juan Ixtayopan y Tulyehualco), que a su vez son parte de la urbanización sur-oriente de la ciudad. Los demás pueblos (11 en total: Villa Milpa Alta, San Pedro Atocpan, San Pablo Oztotepec, San Lorenzo Tlacoyucan, Santa Ana Tlacotenco, San Agustín Ohtenco, San Jerónimo Miacatlán, San Francisco Tecoxpa, San Juan Tepenahuac, San Bartolomé Xicomulco y San Salvador Cuauhtenco) que conforman la Delegación Milpa Alta prácticamente se encuentran separados del área urbana por una barrera geográfica que vendría siendo el Volcán Teuhtli. De ahí que la conformación de muchas de las viviendas en relación a la morfología de los pueblos sea de orden reticular, con ciertas aristas debido a la conformación del territorio en el que se encuentran estos pueblos.
Las preferencias por las construcciones que se derivan de los modelos arquitectónicos de viviendas urbanas, han restado a las características de la vivienda vernácula, lo que hace cada vez más difícil diferenciar una tipología de la vivienda rural en Milpa Alta. No obstante, el interés es lograr establecer los elementos constructivos que, en su momento, resultaron una solución efectiva a las necesidades habitacionales, con una mejor integración al medio natural e incluso constituyeron un elemento estético, que forman parte de una arquitectura rural si bien, en este caso, interesa conocerlos para identificar los elementos rescatables que permitan configurar propuestas más adecuadas a la preservación y defensa de su forma de vida y cultura de los pueblos de Milpa Alta.

\section{FIGURA 4. METODOLOGÍA SOCIO-ESPACIAL.}

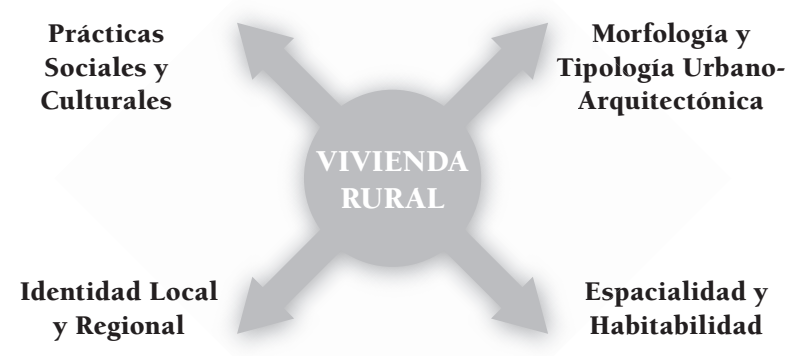

FUENTE: Elaboración Grupal / PMVRS-UNAM-ATH 2011 


\section{Conclusiones}

La vivienda rural en Milpa Alta es un espacio habitacional, construido en el transcurso de 30 años. Como se ha señalado, es posible encontrar la edificación de viviendas por encargo pero resulta el menor de los casos, pues en la generalidad a partir del proceso de autoconstrucción se han edificado la mayoría de las viviendas. Es la solución constructiva que los habitantes han otorgado a sus necesidades habitacionales, considerando los recursos naturales disponibles, las características de las actividades productivas y el sostenimiento de sus tradiciones y cultura como pueblos.

Lo anterior no debe soslayar que las familias resuelven sus necesidades habitacionales con fuertes limitaciones que se traducen en condiciones de vida deterioradas. Como se ha podido constatar, la vivienda rural en Milpa Alta es resultado de un largo proceso de autoconstrucción financiado por sus habitantes, quienes en gran medida perciben ingresos mínimos, sostienen condiciones de vulnerabilidad laboral y carencias en servicios de salud y seguridad social en general; no obstante, sufragan en gran medida los costos de edificación de la vivienda.

Las soluciones de las políticas públicas deben dirigirse en este sentido, sin desconocer las características de la vivienda rural en Milpa Alta, la organización y distribución espacial, los usos de los espacios que responden a su función en la ac- tividad productiva, los arreglos multifamiliares en un mismo predio, la dinámica de poblamiento que se lleva a cabo a través de la herencia familiar de la tierra y el papel que desempeña en la conformación de la vivienda como solución compartida para varias familias, pero no resuelve sus necesidades habitacionales, sobre todo considerando la estructura por edad de la población, predominantemente joven, que formará nuevos hogares, en promedio 4.15 miembros, demandará dotación de servicios, equipamiento, infraestructura y por supuesto la propia vivienda.

Asimismo, la habitabilidad de la vivienda rural también se constituye porque responde a muchos de los comportamientos y actitudes que se manifiestan como pueblos, no sólo de las familias, que actúan como un crisol de valores y de identidades, que conforman un patrón particular de tradiciones y costumbres de las comunidades. Lo cual no se contrapone a la incorporación de materiales, diseños y edificaciones vinculadas con la ciudad, como así lo demuestra la propia solución constructiva de los habitantes de Milpa Alta, por lo que la política pública deberá responder a tales especificidades y características de la vivienda rural, que los habitantes han configurado conforme a sus interacciones sociales, requerimientos y formas de vida como propietarios del territorio.

La vivienda rural construida ha sido una solución efectiva para sus habitantes, la política pública no 
debe subestimar en cuanto a la problemática que representa su edificación en un terreno montañoso, suelo pedregoso y alta pendiente que configura una situación de riesgo e inseguridad para los pobladores, las circunstancias de inhabitabilidad y privaciones materiales de la vivienda, que durante largo tiempo conforman el entorno de las familias deteriorando sus condiciones de vida. Fundamentalmente, si bien la vivienda rural, como parte de un sistema socio-espacial, representa la casa, el territorio e identidad de su forma de vida, para los habitantes no debe condicionarse que la vivienda sea algo particular, que cada familia debe resolver con sus propios recursos.

\section{Bibliografía}

ANUARIO estadístico del DF. México, INEGI. 2004.

BOILS MORALES, Guillermo. Las viviendas en el ámbito rural. Notas, revista de información y análisis (23), 2003. ISSN 1405-6364.

DUHAU, Emilio y GIGLIA, Ángela. Las Reglas del Desorden. Habitar la Metrópoli. México, Siglo XXIUAM. 2008. ISBN:9789682327605.

FLORES Rodríguez, Liliam. La vivienda en México y la población en condiciones de pobreza. México, Centro de Estudios Sociales y de Opinión Pública, Cámara de Diputados. 2009. 72 p. Documento de Trabajo No. 63.
INEGI. Estadísticas del Medio Ambiente del Distrito Federal y Zona Metropolitana. México, INEGI. 2002.

INEGI. I Conteo de Población y Vivienda. México, INEGI. 1995.

INEGI. II Conteo de Población y Vivienda. México, INEGI. 2005.

INEGI. XI Censo de Población y Vivienda. México, INEGI. 1990.

INEGI. XII Censo de Población y Vivienda. México, INEGI. 2000.

JIMÉNEZ ROSAS, Eric Orlando y VERDUZCO CHIRINO, Gemma Luz Silvia. La sintaxis espacial de la vida doméstica. Una comparación urbano-rural. Palapa 4(2): 45-52, julio 2009. ISSN 1870-7483.

SÁNCHEZ QUINTANAR, Concepción. Cambios operativos y funcionales en zona de expansión demográfica. [En línea]. Psicología para América Latina. (7), agosto 2006. ISSN 1870-350X [fecha de consulta: 2 de mayo de 2011]. Disponible en: http:// www.psicolatina.org/Siete/index.html

TAPIA Z, Ricardo. Algunas reflexiones del impacto de la política habitacional chilena en el medio rural. [En línea]. El INVI Opina. Diciembre 2006. [Fecha de consulta: 20 de febrero de 2011]. Disponible en: http://invi.uchilefau.cl/index.php/invi/ vivienda-rural/

revista invi № 73 / Noviembre 2011 / Volumen № 26: 201-223 223 